\title{
EFFECTS OF OVEN DRYING AND POLYDIMETHYLSILOXANE (PDMS) EMULSION COATING ON HEAT-SET PRINTING QUALITY
}

\author{
CEM AYDEMIR, AHMET AKGÜL and DOĞAN TUTAK \\ Marmara University, School of Applied Sciences, Printing Technologies, Istanbul 34722, Turkey \\ ๔orresponding author: C. Aydemir, cemaydemir@marmara.edu.tr
}

Received January 20, 2020

\begin{abstract}
In the heat-set printing drying process, the paper is exposed to oven drier temperatures of about $120-150{ }^{\circ} \mathrm{C}$, so that the solvent in the printed ink film can evaporate. The printed paper is coated with a polydimethyl siloxane (PDMS) emulsion by means of silicone rollers after drying. In this study, the influence of oven drying of the wet ink film and polydimethyl siloxane emulsion coating on the heat-set print quality parameters were investigated. For this purpose, CMYK printed coated papers were passed through a heat-set oven, where the wet ink film was dried, and then coated with PDMS emulsion. Tone value increase (TVI), color CIE $L * a * b *$ and print gloss measurements were made and recorded by taking test print samples from each of the printing, drying and silicone emulsion coating processes. The results were discussed in terms of printability and print quality, and recommendations were made. It has been determined that oven drying reduces half-tone dot values in printed areas on coated papers. In addition, oven drying caused a significant change in black color. PDMS emulsion coating reduced print gloss, similarly to oven drying.
\end{abstract}

Keywords: heat-set printing, oven drying, hot air dryer, silicone oil emulsion, polydimethyl siloxane

\section{INTRODUCTION}

Heat-set web offset (HSWO) printing is typically used for magazines, catalogs, inserts and other medium-to-high volume and medium-tohigh quality production runs. ${ }^{1}$ Heat-set means an offset printing process where evaporation takes place in an oven where hot air is used to heat the printed material. ${ }^{2}$ This subset of web-fed offset printing uses inks that dry by evaporation in a dryer typically positioned just next to the printing units. ${ }^{1}$ The local temperature rise in the dryer causes the ink solvent to evaporate. ${ }^{3}$

The visual quality of print results is substantially based on the optimal level of ink merging with a substrate, particularly on the drying and stabilization processes of ink on the substrate. ${ }^{4}$ Three basic mechanisms control ink behavior on paper surfaces. These mechanisms are penetration, evaporation and oxidation. ${ }^{5}$ Most offset inks do not dry by evaporation, but rather by oxidation or absorption in the paper. Heat set inks are an exception. ${ }^{2}$ They dry out chiefly by evaporation. ${ }^{6}$ They dry mainly physically by evaporation of the (high boiling) component of the solvent. In this system, the printed paper passes through the heat-set dryer (about 0.7-1.0 seconds) at the exit of the final printing unit to prevent contamination of the fresh ink and to execute precise finishing operations, such as folding-cutting. The hot air flowing from the burners in the drying oven heats the paper path. Thus, the wet ink film is dried. This is accomplished with hot air drying at temperatures between $120-150{ }^{\circ} \mathrm{C}$ (depending on the paper grade and ink coverage). The most important parameters influencing the drying process are dryer air temperature, temperature profile and ink amount. For coated papers, the coat weight also has a significant effect. Unexpectedly, a considerable amount of solvent evaporates simultaneously with moisture at the early stages of drying. For normal drying conditions, some solvent and moisture remain in the paper structure. ${ }^{7}$

The air required to dry the ink on the paper path is supplied with radial reactive blowers. Ventilators transfer the air through the air duct system to the reactive blowers. The air exiting the reaction blowers hits the paper path at a speed of about $200 \mathrm{~km} / \mathrm{h}$ and is then directed back to the fans, while the flow velocity is controlled. As the paper passes through the oven, the oil-based solvents in the ink reach a "flashpoint" or 
evaporation point. $70-85 \%$ of the mineral oil in the printed ink film is evaporated in the heat-set dryer. Waxes, resins, pigments and about 20\% mineral oil remain on the paper surface. After the paper path has left the dryer, the remaining adhesive ink layer is drawn by the surface forces of the paper. The paper then passes through chilling rollers, where the waxes and resins cool down and solidify, or set. ${ }^{8}$ Thus, the pigment component in the resin remaining on the paper surface is solidified by an oxidationpolymerization reaction. This process of printing yields a cleaner more upscale product.

The triple burners that can be managed separately in the drying ovens can bring about problems of wear, contamination and carbonization in the folding unit of the product and after printing, as the amount of the evaporated mineral oil in the printed ink film becomes lower when the temperature is kept low. The excess non-evaporating mineral oil concentration, caused by the inadequacy of the dryer, on the surface of the paper leads to the formation of an oil film on the cooling rollers. This causes contamination in the printed areas, makes the ink on the paper become sticky and contaminates the folding hoppers and the unit. If the solvent vapors are withdrawn from the printed material before it cools, then undesired condensation is avoided. ${ }^{9}$ For this reason, the temperature of the drying oven must be precisely adjusted according to the weight of the paper and the density of the ink. However, some characteristics of the paper and some print quality parameters may change even when the oven heat is at the ideal levels for ink drying. The reduction in the relative humidity of the paper during the drying process is tried to be prevented by means of re-moisturizing units in the paper path cooling groups.

The adhesive ink layer on paper (having a temperature above $90{ }^{\circ} \mathrm{C}$ ) that has just been removed from the drying oven can be reduced to a temperature below $30{ }^{\circ} \mathrm{C}$ by passing through the rollers of the cooling group, so that it can be easily folded off by the folding apparatus. Thus, the printed areas, which are soft and sticky at high temperatures, become hardened by lowering the heat to a low temperature and prevent paper contamination.

The paper from the cooling rollers is coated with emulsions of polydimethyl siloxane and diverse additives in water at the final stage of the heat-set printing process. The chemical formula for PDMS is $\mathrm{CH}_{3}\left[\mathrm{Si}\left(\mathrm{CH}_{3}\right)_{2} \mathrm{O}\right]_{\mathrm{n}} \mathrm{Si}\left(\mathrm{CH}_{3}\right)_{3}$ (Fig. 1), where $\mathrm{n}$ is the number of repeating monomer $\left[\mathrm{SiO}\left(\mathrm{CH}_{3}\right)_{2}\right]$ units. ${ }^{10}$ Industrial synthesis can begin from dimethyldichlorosilane and water by the following net reaction:

$$
\begin{aligned}
& n \mathrm{Si}\left(\mathrm{CH}_{3}\right)_{2} \mathrm{Cl}_{2}+(\mathrm{n}+1) \mathrm{H}_{2} \mathrm{O} \rightarrow \mathrm{HO}\left[-\mathrm{Si}\left(\mathrm{CH}_{3}\right)_{2} \mathrm{O}\right. \\
& -]_{n} \mathrm{H}+2 n \mathrm{HCI}
\end{aligned}
$$

Polydimethyl siloxane and diverse additives are converted into emulsion in water and thus a silicone oil/water emulsion film is formed. In heat-set web offset press applications, watersilicone oil emulsions are used to prevent ink smearing, improve print gloss, protect the ink on the paper, for an anti-static effect, anti-mist effect, water-repellency and flattening of the printed matter, and to increase the strength of the paper surface against breaking and tearing. ${ }^{11}$

The exposure of printed paper to high drier temperature and to silicone emulsion film coating can change the dimensional, optical, superficial and strength properties of the paper. Also, circular half-tone dot values can change color, print gloss, friction strength etc. of the ink film. In this study, the effects of oven drying and silicone emulsion film coating on print quality parameters at ideal temperature after heat-set web offset printing were examined.

\section{EXPERIMENTAL}

\section{Testing and printing}

A color test chart was prepared to measure the print quality parameters of the printed sheets in the entire production process. In this test chart, four color "solid tone" fields were used to measure changes in the colorimetrical tone value and print gloss values, and the original tone value fields from 0 to 100 were used to measure circular half-tone dot value.

For the test prints, UPM $86 \mathrm{~cm} 90 \mathrm{~g} / \mathrm{m}^{2}$ matte and gloss coated papers, with different surface structures, were used (Table 1).

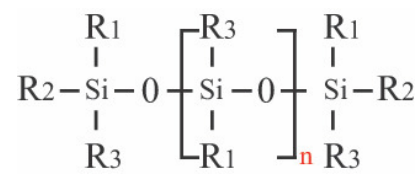

Figure 1: Chemical structure of a PDMS molecule 
Printing

Table 1

Specifications of the papers

\begin{tabular}{llccc}
\hline Properties & Standards & Units & $\begin{array}{c}\text { Matte coated } \\
\text { paper }\end{array}$ & $\begin{array}{c}\text { Gloss coated } \\
\text { paper }\end{array}$ \\
\hline Grammage & ISO 536 & $\left(\mathrm{g} / \mathrm{m}^{2}\right)$ & 90 & 90 \\
Bulk & ISO 534:1995 & $\left(\mathrm{cm}^{3} / \mathrm{g}\right)$ & 0.82 & 0.77 \\
Whiteness CIE D65/10 & ISO 11475 & $(\%)$ & 84.50 & 83.55 \\
Brightness D65/10 & ISO 2470-2 & $(\%)$ & 82.64 & 78.36 \\
Opacity with D65/10 & ISO 2471 & $(\%)$ & 94.5 & 94 \\
Gloss TAPPI 75 & TAPPI T480 & $(\%)$ & 29.9 & 66.9 \\
Surface roughness (PPS 1.0) & ISO 8791-4 & $(\mu \mathrm{m})$ & 1.33 & 0.82 \\
Ash content & TAPPI T211 & $(\%)$ & 38.09 & 38.2 \\
Surface energy & ASTM D5946 & $\left(\mathrm{mJ} / \mathrm{m}^{2}\right)$ & 52.6 & 50.6 \\
\hline
\end{tabular}

Coated papers, with a width of $860 \mathrm{~mm}$, were conditioned in accordance with Tappi T402 om-88 method at $23 \pm 1{ }^{\circ} \mathrm{C}$ and $50 \pm 2 \%$ relative humidity, for at least 24 hours before testing. "Sun Chemical SunFasion" HS series process CMYK colours ink set (DIN ISO 2846-1:2017) was used in the heat-set web offset lithographic printing process. The $350 \times 500 \mathrm{~mm}$ color test chart was used as the test print area. This test chart has solid tone and half-tone areas for each ink color. Test printing was done in accordance with FOGRA 51 standard on a GOSS-M600 (max. web width: $965 \mathrm{~mm}$ ) Web Offset printing machine at constant printing speed $(7.2 \mathrm{~m} / \mathrm{s})$. During the printing process, Flint Group dayGraphica 5000 blanket, with compressible layer technology, AGFA Thermostar P970 thermal printing plate and TOYO PF-21000 dampening solution, designed for alcohol-free printing, with a conductivity of $9 \mathrm{~m} . \mathrm{S} / \mathrm{cm}$ and a $\mathrm{pH}$ value of $4.80 \pm 0.2$, were used.

Prints were made with KCMY color. Then, $130{ }^{\circ} \mathrm{C}$ web drier temperatures were applied to all the paper surfaces in a heat-set drying oven to dry the wet ink films. After the ink film was dried, a standard viscosity silicone emulsion (Flint Group Varn Pro-Web Platinum Plus) was applied as coating, with a standard silicone applicator setting.

After the printing process was completed, CIE $L * a * b *$ (CIELAB) measurements from the "solid tone" areas of the print test chart and dot gain measurements from the "halftone dot" areas were made for the CMYK colors, with an X-Rite eXact ${ }^{\mathrm{TM}}$ Spectrophotometer. Measurements were performed according to ISO 12647-2: 2013 standard, with D50 illuminant, $2^{\circ}$ observer, $0 / 45$ or $45 / 0$ geometry, black backing and M1 mode measurement features. Print gloss values of ink films were measured at $60^{\circ}$ geometry in accordance with ISO 2813:2014 standard with a glossmeter from BYK-Gardner $\mathrm{GmbH}$, Geretstried, Germany. On average, 40 replicates were made per test. The results of these 40 test print samples were then averaged.

In order to determine the effect of oven drying and polydimethyl siloxane emulsion coating on ink light fastness, all the samples that had been printed, dried and coated with silicone emulsion were subjected to individual light fastness tests in accordance with British Standard (BS) 4321, using a Solarbox 1500e tester. All CMYK color and print gloss values were remeasured after fading and the results were recorded. Ink color differences ( $\Delta E^{*}{ }_{a b}$ CIE 1976), before and after fading, and print gloss variations were also calculated and tabulated.

\section{RESULTS AND DISCUSSION Print quality}

Heat-set test printing operations were carried out in accordance with 12647-2:2013 standard with mineral oil-based CMYK color process inks that meet the DIN ISO 2846-1 standard. Solid tone value, CIE $L^{*} a^{*} b^{*}$ and print gloss measurements were made for every color on the test chart, at every stage of printing, oven drying and silicone emulsion coating processes, on matte coated and gloss coated papers in the production process. Half-tone dot, color and gloss value changes were calculated separately and the results are presented. In addition, the color difference $\left(\Delta E^{*}{ }_{a b}\right)$ and print gloss changes, before and after fading, were calculated for both color and brightness values by the fading test for each color. All the measurements are shown in the tables and graphs below.

\section{Dot gain/TVI (tone value increase)}

When a half-tone dot is transferred from the film via the plate and the blanket to the printing substrate, several factors may change its geometric size, and thus its half-tone value as well.

Dot gain is one of the most discussed variables in offset printing and is a measure of the difference between the half-tone dot size (final tone value) of the printed image and the half-tone dot size (original tone value) specified by the source file. Dot gain is a measurable, predictable 
and controllable effect of the printing process. ${ }^{12-14}$

As the half-tone dots move through each stage of the printing process they are altered slightly. To accurately produce four color images, dot gain must be controlled. ${ }^{11}$ If not taken into account, the result is a printed image that looks darker than intended. A scanned image that looks fine on screen may be too dark for printing. ${ }^{13}$ Dot gain in screened densities also causes huge changes in hue and saturation. ${ }^{15}$

The amount of dot gain is influenced by different factors. Especially, the type of press (offset, flexo, gravure, etc.), printing process, surface characterization of paper, the type of ink and ink thickness have a great influence on the dot gain. ${ }^{15}$ In this part of the study, the half-tone micro dot values for all the process colors of all the samples obtained from the heat-set printing processes were measured with an X-Rite eXact ${ }^{\mathrm{TM}}$ Spectrophotometer and the results are given in Figure 2.

The oven drying process applied to the ink film after printing has significantly reduced the half-tone point values of black, cyan and magenta color, but not of yellow (Fig. 2). The PMDS emulsion coating process (after the oven drying process) did not cause a significant difference in

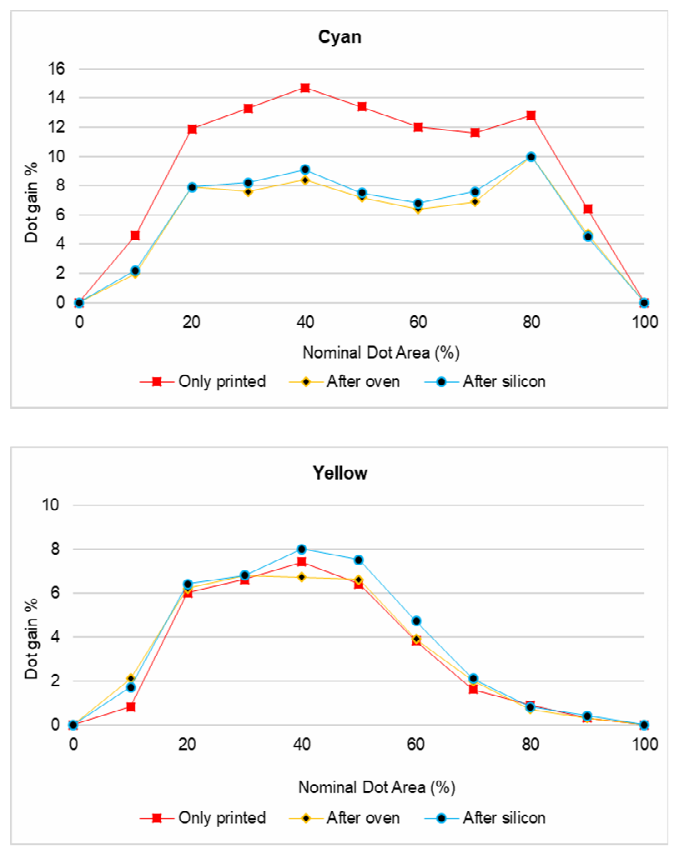

the half-tone point values. This indicates that the oven drying process reduces the dot value on matte coated paper, and the PMDS emulsion coating has no effect on the dot value in the printed image.

On the gloss coated papers, there was no significant change in the black ink film tone values during the post-printing heat-set processes, while the yellow color tone values increased and the magenta and cyan color dot gain values decreased (Fig. 3). These results showed that oven drying can change especially print mid-tone values, depending on the color and paper type. The PDMS emulsion coating, which took place after the oven drying process, did not cause significant changes in the half-tone dot values (after oven drying).

\section{CIE $L * a * b *$ colour differences $\left(\Delta E^{*}{ }_{a b}\right)$}

The CIE $L * a * b *$ colour space is a threedimensional, spherical model. ${ }^{16}$ In this model, $L^{*}$ defines lightness, $a^{*}$ denotes the red/green value and $b^{*}$ - the yellow/blue value. $\Delta E^{*}{ }_{a b}$ is defined as the difference between two colors in an $L^{*} a^{*} b^{*}$ color space and represents the difference in each of the $L, a$ and $b$ readings compared to the initial readings. ${ }^{17}$
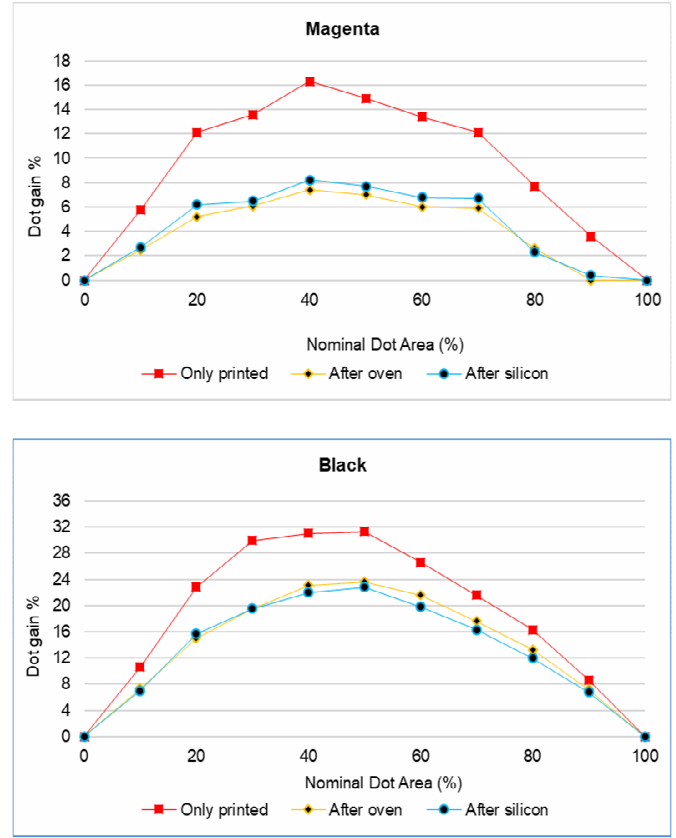

Figure 2: Change in half-tone dot values printed on matte coated paper 

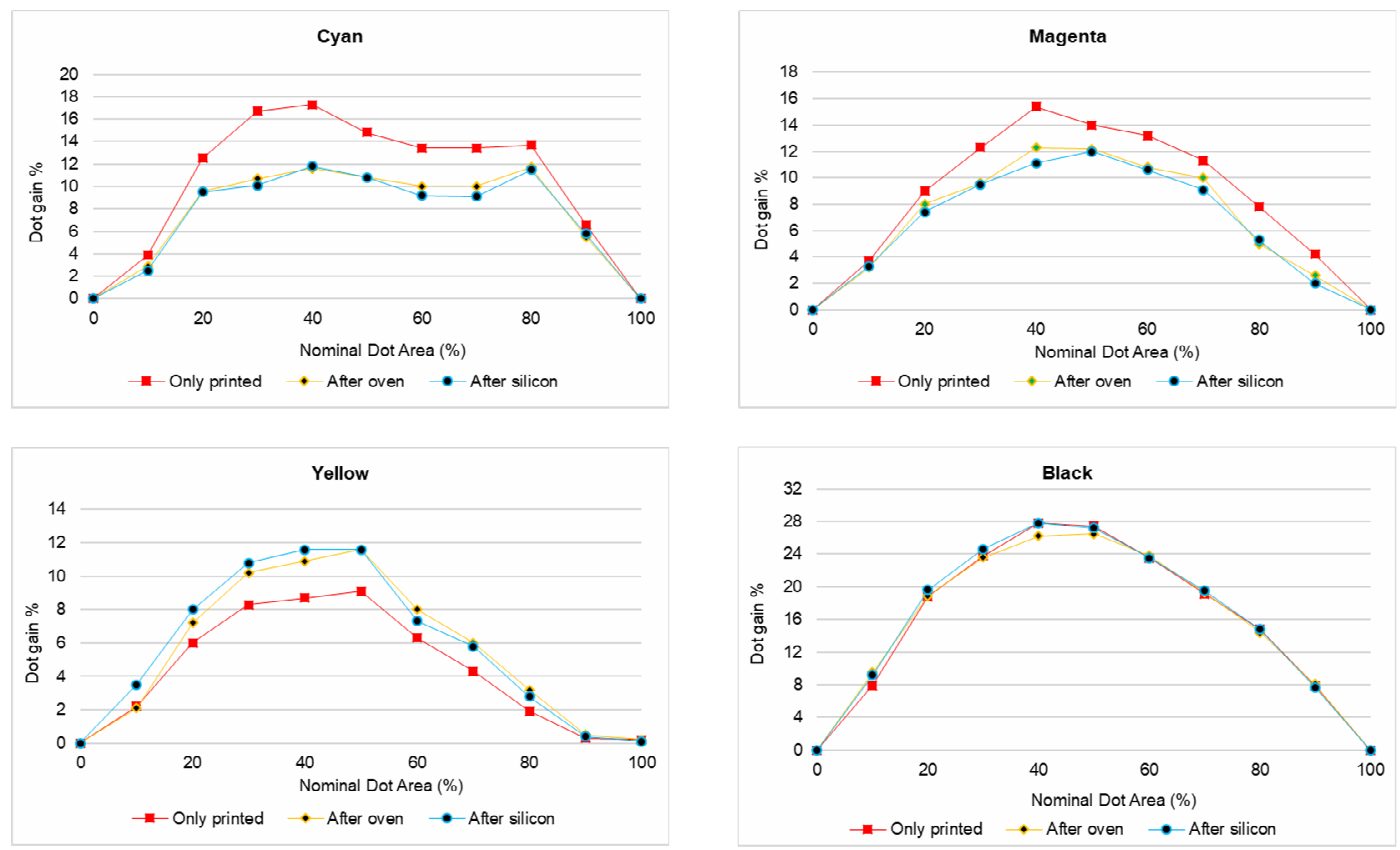

Figure 3: Change in printed half-tone dot values on gloss coated paper

The CIE $1976 L^{*} a^{*} b^{*}$ colour difference, $\Delta E^{*}$ ab , between two colour stimuli is calculated as the Euclidean distance between two points, representing them in the space (Eq. (1)):

$\Delta E_{a b}^{*}=\left[\left(\Delta L^{*}\right)^{2}+\left(\Delta a^{*}\right)^{2}+\left(\Delta b^{*}\right)^{2}\right]^{1 / 2}$

where $\Delta L^{*}, \Delta a^{*}$ and $\Delta b^{*}$ are differences in $L^{*}, a^{*}$ and $b^{*}$ values between the specimen color and the target color.

Colour change expressed as delta $\mathrm{E}(\Delta \mathrm{E})$ is important for printing, and it is normally required to be below 1.0, since it is the colour difference that the human eye cannot detect. ${ }^{18}$ In accordance with their perceptibility, the colour location differences can be classified as follows:

$\Delta E^{*}{ }_{a b}$ between 0 and $1=$ in general, this deviation cannot be perceived;

$\Delta E^{*}{ }_{a b}$ between 1 and $2=$ very small deviation, only perceivable by an experienced eye;

$\Delta E^{*}{ }_{a b}$ between 2 and $3.5=$ medium deviation, perceivable even by an unexperienced eye;

$\Delta E^{*}{ }_{a b}$ between 3.5 and $5=$ large deviation;

$\Delta E^{*}{ }_{a b}$ exceeding $5=$ massive deviation. ${ }^{19}$

In this study, color CIE $L^{*} a * b *$ measurements were made from process-ink set single-print solid areas after printing, oven drying and polydimethyl siloxane emulsion application in accordance with ISO 13655. Then, the difference $\left(\Delta E^{*}{ }_{a b}\right)$ of the CMYK colors obtained in these production steps from the previous process $\left(\Delta E^{*}{ }_{a b}\right)$ was calculated in accordance with Equation (1).
On the matte and glossy coated paper surfaces, after the oven drying process, the least color change occurred in cyan. After PDMS emulsion coating, the minimum color change was seen in black on the matte coated paper and yellow on the gloss coated paper. In both types of paper, the color change effect of the oven drying process was greater than that of the silicone oil application (Table 2). The reason for this is thought to be due to the evaporation of the mineral oil from the ink content due to the influence of the oven temperature and thus the increase in the pigment content in the ink film.

As regards the coated papers, it was determined that the highest color change, after all the heat-set processes, was seen in the black ink film. For the black color, large deviation in matte paper and medium deviation in glossy paper were found (Table 3).

In general, oven drying and PDMS emulsion coating did not cause changes perceptible by the human eye in magenta, cyan and yellow (Table 3 ). However, the results show that the changes in black may negatively affect all the print colors and the print outcome.

\section{Print gloss contrast}

The quality of an ink film can be described by optical properties, such as gloss and optical color density. ${ }^{20}$ Gloss is a visual impression resulting 
from surface evaluation and is one of the most significant qualities of all print products. Smooth and highly polished surfaces reflect images distinctly. ${ }^{21}$

When an ink appears glossy, sufficient ink vehicle has filmed the surface of the paper to provide a covering layer for pigment particles in the ink. When an ink appears dull, sufficient vehicle has been drained from the surface, so that the irregularities of individual pigment particles and the paper surface are not covered with a vehicle film thick enough to create gloss. ${ }^{22}$

In this study, the print gloss values of ink film surfaces, dried ink film and CMYK ink film coated with PDMS emulsion were measured separately, with $60^{\circ}$ geometry, according to ISO 2813:2014 standard, using a glossmeter from BYK-Gardner GmbH.

Table 2

Ink color difference in heat-set compared to that of previous production processes

\begin{tabular}{lccccccccc}
\hline \multirow{2}{*}{ Process } & \multicolumn{4}{c}{ Colour difference $\left(\Delta E^{*}{ }_{a b}\right)$} \\
\cline { 2 - 9 } & \multicolumn{3}{c}{ Matte coated paper } & \multicolumn{4}{c}{ Gloss coated paper } \\
\cline { 2 - 9 } & Black & Magenta & Cyan & Yellow & Black & Magenta & Cyan & Yellow \\
\hline $\begin{array}{l}\text { After oven drying } \\
\text { (compared to printing) }\end{array}$ & 4.036 & 0.804 & 0.062 & 0.135 & 1.989 & 0.746 & 0.273 & 0.931 \\
$\begin{array}{l}\text { After PMDS emulsion coating } \\
\text { (compared to oven drying) }\end{array}$ & 0.093 & 0.300 & 1.238 & 0.249 & 0.206 & 0.272 & 0.215 & 0.176 \\
\hline
\end{tabular}

Table 3

Total ink color difference in final products of heat-set printing

\begin{tabular}{cccccccc}
\hline \multicolumn{7}{c}{ Colour difference $\left(\Delta E^{*}{ }_{a b}\right)$} \\
\hline Black & Magenta & Cyan & Yellow & Black & Magenta & Cyan & Yellow \\
\hline 3.964 & 0.532 & 1.268 & 0.187 & 2.065 & 0.731 & 0.112 & 0.763 \\
\hline
\end{tabular}

Table 4

Print gloss $\left(60^{\circ}\right)$ values for heat-set printing process

\begin{tabular}{lcccccccc}
\hline \multirow{2}{*}{ Process } & \multicolumn{7}{c}{ Print gloss $\left(60^{\circ}\right)$} \\
\cline { 2 - 8 } & \multicolumn{7}{c}{ Matte coated paper } & \multicolumn{4}{c}{ Gloss coated paper } \\
\cline { 2 - 8 } & Black & Magenta & Cyan & Yellow & Black & Magenta & Cyan & Yellow \\
\hline Printed & 28.4 & 29.23 & 27.88 & 33.34 & 50.41 & 46.63 & 46.24 & 57.26 \\
After oven drying & 28.3 & 27.72 & 27.75 & 32.87 & 46.05 & 44.95 & 42.18 & 48.9 \\
After silicone & 28.08 & 22.92 & 24.32 & 31.62 & 44.45 & 43.49 & 41.23 & 48.8 \\
emulsion coating & 28.92 & & & & & & & \\
\hline
\end{tabular}

The process of oven drying showed a decreasing effect in the brightness of the process colors on glossy coated paper, but no significant change on matte coated paper. This situation shows the effect of paper surface characteristics on print production processes, considering that all the print production operations were carried out under the same conditions. Silicone oil emulsion coating reduced the print gloss of all process (CMYK) colors on both paper types (Table 4).

\section{Light fastness}

The lightfastness of prints is the ability to retain their color strength and resist fading upon exposure to sunlight. ${ }^{23}$ In this study, the light fastness test was applied to all the test samples that were printed, oven dried, and coated with the PDMS emulsion film to determine the effect of the heat-set web processes on the color strength of the printed ink. The color CIE $L^{*} a^{*} b^{*}$ and the color print gloss values of these test samples, before and after fading, were measured separately to reveal the differences in print gloss and color change $\left(\Delta E^{*}{ }_{a b}\right)$. Thus, the effect of the heat-set web offset printing processes on color strength was determined (Tables 5, 6).

After the light fastness test applied separately for each heat-set web process on matte and glossy coated papers, changes were observed in yellow, cyan and magenta color inks. The variation 
tolerance values in these colors after fading do not conform to ISO $12647-2$ standards $\left(\Delta E^{*}{ }_{a b}<4\right)$ and were at visually noticeable levels. Especially yellow faded almost completely. The least change, on both paper types, was seen in black ink color. After the light fastness test, the change in the magenta color ink film was more pronounced on the coated glossy paper surface.

Table 5

Color difference $\left(\Delta E^{*}{ }_{a b}\right)$ values after light fastness test

\begin{tabular}{lcccccccc}
\hline & \multicolumn{8}{c}{ Colour difference $\left(\Delta E^{*}{ }_{a b}\right)$} \\
\cline { 2 - 9 } Process & Black & Magenta coated paper & Cyan & Yellow & Black & Magenta & Cyan & Yellow \\
\hline Printed & 2.28 & 3.09 & 4.13 & 16.78 & 1.49 & 8.54 & 11.1 & 57.06 \\
After oven drying & 1.88 & 4.8 & 11.71 & 12.81 & 2.03 & 8.28 & 10.71 & 62 \\
After PMDS & 2.07 & 5.06 & 6.62 & 20.56 & 2.12 & 7.45 & 11.54 & 63.29 \\
emulsion coating & 2.07 & & & &
\end{tabular}

Table 6

Print gloss values after light fastness test

\begin{tabular}{lcccccccc}
\hline \multirow{2}{*}{ Process } & \multicolumn{8}{c}{ Print gloss $\left(60^{\circ}\right)$} \\
\cline { 2 - 9 } & \multicolumn{7}{c}{ Matte coated paper } & \multicolumn{5}{c}{ Gloss coated paper } \\
\cline { 2 - 9 } & Black & Magenta & Cyan & Yellow & Black & Magenta & Cyan & Yellow \\
\hline Printed & 22.5 & 23.6 & 21.4 & 26.1 & 43.7 & 41.7 & 38.5 & 48.9 \\
After oven drying & 23.2 & 23.1 & 19.5 & 26 & 41.2 & 38.8 & 34.8 & 41.4 \\
After PMDS & 23.2 & 20 & 19.4 & 24.1 & 40.4 & 38.3 & 33.1 & 41.3 \\
emulsion coating & 23.2 & & & & &
\end{tabular}

In general, the most significant change in the colors after the heat-set web processes occurred on glossy coated paper surfaces. As a result, the printing substrate is as effective as the ink when considering the resistance of colors to light. In addition, there was a change surpassing the standards in cyan after oven drying on coated papers. Oven drying and PDMS emulsion coating adversely affected the printed ink films in terms of color strength.

After the light fastness test, it has been determined that the application of oven drying and silicon emulsion coating in the heat-set reduces the ink printing gloss, compared to that of only printed samples. In this case, oven drying and PMDS emulsion coating do not contribute to protecting the color gloss and to increasing the resistance to fading.

\section{CONCLUSION}

In this work, the effects of oven drying and silicone emulsion film coating on print quality parameters in heat-set web offset printing were examined. The conclusions are summed up below.

- It has been determined that, in general, both oven drying and PDMS emulsion film coating have changed the printing color, tone value and print gloss value, when the heat-set web offset printing processes are evaluated. This effect varied as a function of the paper surface characteristics and the printing color. For this reason, paper characteristics and ink color are important factors to be considered and appropriate planning should be done in order to obtain the desired values of print quality parameters at the end of the heat-set web production process chain.

- It should also be emphasized that oven drying and PDMS emulsion coating in heat-set web printing will cause significant changes in printing colors and printing outcome, and will increase or decrease the tone value, depending on the ink color selection and paper surface characteristics. In order to minimize this negative effect, the print characteristic curve or the absolute tone value increase should be considered in the generation of the color separation data. Depending on the printing characteristics, the size of the dots should be changed, and dot tone percentages and color contrast curve adjustments should be made. In addition, separate \% increase or decrease in tone value adjustments for each color should be made in the table of CtP (computer to plate) RIP unit, considering ink thickness, the printing substrate and, especially, the printing process effects. Only in this way, tone 
values conforming to the standard tolerances of ISO 12647-2 could be achieved first on printing plate and then on paper.

- Since the changes in black color after oven drying and PDMS emulsion coating result in variations that do not conform to the ISO 12647-2 standard tolerance values, this situation should be taken into account in the pre-printing tone setting.

- Production planning for products requiring high print gloss should be done, depending on the printing ink color and paper surface characteristics, considering the print gloss effects of oven drying and PDMS emulsion coating in heat-set web offset printings.

- The PDMS emulsion application in the heat-set reduces the glossiness before and after the light fastness test. To eliminate this negative effect, hybrid silicone oils, synthetic bases, waxes, optical brighteners can be added to the silicone emulsion. A new type of silicone technology can be developed. It should also be noted that the migration values of these silicone emulsions should also be low, considering their environmental and health impact. Silicon films with low migration, but efficient and with an effective concentration should be obtained on the paper surface.

- For situations that require light fastness, when the oven drying cannot be renounced, considering that oven drying and PDMS emulsion coating reduce color strength and print gloss, silicone oil coating should be applied, especially, for products intended for outdoor uses.

ACKNOWLEDGEMENT: This work is based on the $\mathrm{PhD}$ thesis entitled "Determination of the Ideal Drying Temperature of Heat-set Web Offset Inks by Vapor Pressure Method". The authors would like to thank the Sabah Newspaper Printing, where all the printing tests of this study were carried out.

\section{REFERENCES}

1 C. Aydemir, Ph.D. Thesis, Marmara University, Institute of Natural and Applied Sciences, Turkey, 1999

2 EGTEI, Heatset-Offset, 2005, pp. 3, https://ec.europa.eu/environment/archives/air/stationar y/solvents/activities/pdf/d001_synopsys_sheet_heatset _offset.pdf

$\overline{3}$ P. Gerstner, T. Grönblom and P. A. C. Gane, J. Print Media Technol. Res., 37, 135 (2010), http://jpmtr.org/Advances-Vol-37(2010)_online.pdf (accessed January 2, 2020)
4 C. Aydemir, A. Karademir, S. İmamoğlu, B. N. Altay, P. D. Fleming et al., Cellulose Chem. Technol., 53, 787 (2019) https://doi.org/10.35812/CelluloseChemTechnol.2019. 53.77

5 C. Aydemir, Z. Özomay, A. Karademir and E. A. Kandırmaz, Sci. Eng. Compos. Mater., 20, 141 (2013), https://doi.org/10.1515/secm-2012-0070

6 J. Y. Hung and J. A. Forst, Dry. Technol., 9, 501 (1991), https://doi.org/10.1080/07373939108916678

K. Koivumaki and E. K. O. Héllen, in Procs. $36^{\text {th }}$ International Research Conference of Iarigai, Stockholm, Sweden, September 13-16, 2009, pp. $267-$ 271 http://jpmtr.org/Advances-Vol36(2009)_online.pdf

8 Sappi, Web Heatset Ink Chill-Pick and Solvent Streaking, Sappi Fine Paper North America, 2012, https://cdn-s3.sappi.com/s3fs-public/sappietc/SappiPrinter-Tech-Tips-Web-Chill-Pick-SolventStreaking.pdf

9 R. A. Daane, US4462169A, 1982, https://patents.google.com/patent/US4462169A/en

10 M. Holban and A. M. Grumezescu, "Materials for Biomedical Engineering: Hydrogels and Polymerbased Scaffolds", Elsevier, 2019, pp. 491

11 GOVI, Heatset printing, http://www.govi.com/emulsions/siliconeemulsions/heatset-printing

12 L. Yang and N. Lundström, Nord Pulp. Pap. Res. J., 22, 388 (2018), https://doi.org/10.3183/npprj-200722-03-p388-393

13 M. Namedanian, L. G. Coppel, M. Neuman, S. Gooran, P. Edström et al., J. Imaging Sci. Techn., 57, 20504-1 (2013), https://doi.org/10.2352/J.ImagingSci.Technol.2013.57. 2.020504

14 R. Klein, G. Meder, O. Höhne and J. Tomaszewski, in Procs. $40^{\text {th }}$ International Research Conference of Iarigai, Chemnitz, Germany, September 08-11, 2013, pp. 205-217, http://jpmtr.org/Advances-Vol40(2013)_online.pdf

15 Esko, "Dot gain - User Guide", 2014, https://docs.esko.com/docs/en-

us/curvepilot/14/userguide/home.html?q=enus/common/cup/concept/c_aa1038777.html

16 P. Nussbaum, Doctoral Dissertation, Department of Informatics, University of Oslo, Norway, 2010

17 "X-Rite Pantone, A Guide to Understanding Color", 2016, p. 1-39, https://www.xrite.com//media/xrite/files/whitepaper_pdfs/110

001_a_guide_to_understanding_color_communication/ 110-001_understand_color_en.pdf

18 C. Aydemir, Sci. Eng. Compos Mater., 23, 565 (2016), https://doi.org/10.1515/secm-2013-0266

19 L. S. Lade, IJEMS, 4, 1 (2013), http://scienceandnature.org/IJEMS-Vol4(1)Jan2013/IJEMS_V4(1)1.pdf

${ }^{20}$ C. Aydemir, S. Yenidoğan, A. Karademir and E. A. Kandirmaz, J. Appl. Biomater. Func., 16, 137 (2018), 
https://doi.org/10.1177/2280800018764761

21 C. Aydemir, S. Yenidoğan, A. Karademir and E. A. Kandirmaz, Mater. Manuf. Process., 32, 1310 (2017), https://doi.org/10.1080/10426914.2017.1279323

22 S. D. Warren, Technical Bulletin No. 1, 1988, p. 117, https://cdn-s3.sappi.com/s3fs-public/sappietc/SD-
Warren-Bulletin-Ghosting.pdf

23 I. Bates, I. Zjakić and M. Milković, Tehn. Vjesn., 18 , 349 (2011), https://pdfs.semanticscholar.org/c0a0/af2fb032c4299d 3ef59dd147fc61c7982626.pdf 